\title{
Thromboembolierisiko bei Verhütung mit Spirale oder Implantat am geringsten
}

Fragestellung: Wie hoch ist die Gefahr von Thromboembolien für Frauen mit Typ-1- und Typ-2-Diabetes bei hormonaler Kontrazeption?

Hintergrund: Nahezu drei von vier Frauen mit Diabetes im gebärfähigen Alter leben ohne Kontrazeption. Da wundert es nicht, dass zwei von drei Schwangerschaften bei diesen Frauen ungeplant sind. Für Kontrazeption spricht, dass bei sorgfältig geplanten Schwangerschaften Hyperglykämien und teratogene Folgen besser vermeidbar sind. Andererseits fürchtet man das Thromboembolierisiko, das bei Diabetikern spontan erhöht ist und durch hormonale Kontrazeption noch verstärkt werden kann.

Patienten und Methoden: Die Daten stammen aus einer amerikanischen Datenbank, die anonymisiert demografische und sozioökonomische Fakten, die Ausgabe von Arzneimitteln nach Art und Dosis, Laborwerte, ärztliche Diagnosen und Klinikbehandlungen enthält. Innerhalb von 10 Jahren konnten 47 Millionen Personen registriert werden. In den Jahren 2002 - 2011 befanden sich darunter 146.080 Typ-1- und Typ-2-Diabetikerinnen im Alter von 14 - 44 Jahren mit Verordnungen einer Kontrazeption.

Ergebnisse: Die Mehrzahl der Frauen im gebärfähigen Alter (72 \%) hat zu keinem Zeitpunkt irgendeine hormonelle Verhütung praktiziert. Unter Kontrazeption traten insgesamt 3.012

\section{Originalie}

O'Brien S H, Koch T, Vesely S K und Schwarz E B. Hormonal Contraception and Risk of Thromboembolism in Wornen With Diabetes. Diabetes Care. 2017;40:233-8 thromboembolische Ereignisse auf, d.h. Herzinfarkte, Schlaganfälle oder Venenthrombosen. Daraus errechnet sich ein nur niedriges absolutes ThromboembolieGesamtrisiko zwischen 10 - 16 Ereignissen pro 1.000 Patientenjahre. Die höchste Komplikationsrate

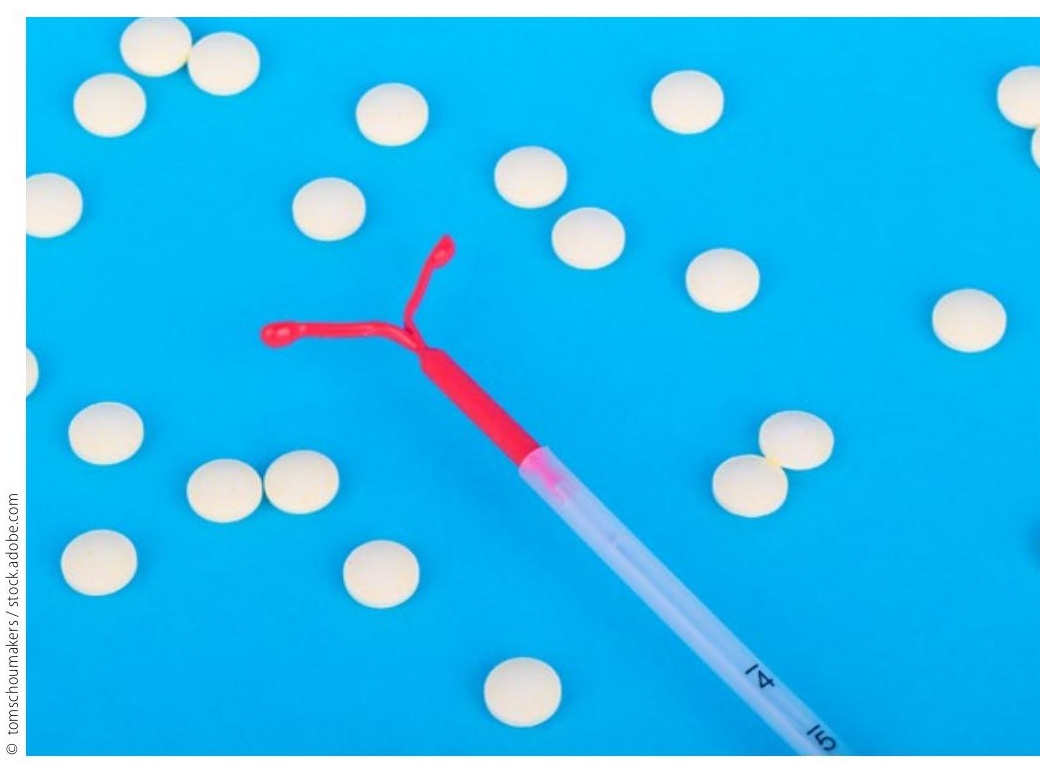

Familienplanung macht bei Diabetes besonders Sinn, um einem Kind den bestmöglichen Start zu geben. Die Pille ist im Vergleich zur Spirale in puncto Thrombenbildung bei Mama aber ungünstiger.

fanden die Autoren bei der Kontrazeption mit dem Hormonpflaster und dem Vaginalring (16,4 bzw. 13,5 pro 1000 Patienten), die geringste mit der intrauterinen und - bei nur kleiner Fallzahl - subdermalen Methode (3,4 und 0 pro 1000). Bei allen Ergebnissen wurden mögliche Einflussfaktoren wie Alter, diabetische Komplikationen, Hyperlipidämie, Hypertonie, Rauchen und maligne Erkrankungen berücksichtigt.

Schlussfolgerung: Die intrauterine und die subdermale hormonelle Kontrazeption sind für Diabetikerinnen besonders geeignete Methoden mit niedrigem Thromboembolierisiko.

\section{- Kommentar von Prof. Dr. med. Heinrich Holzgreve}

\section{Implantation sticht Tabletteneinnahme}

Das Thromboembolierisiko ist bei Diabetikern glücklicherweise gering, auch unter hormonaler Kontrazeption. Trotzdem zeigt diese Studie für die einzelnen Varianten der hormonellen Kontrazeption Unterschiede um den Faktor 15 - 20. Die Autoren propagieren die intrauterine und subdermale hormonale Kontrazeption aus guten Gründen. Einerseits ist das Thromboembolie-Risiko am geringsten. Andererseits handelt es sich um zwei Methoden, die implantiert werden, jahrelang wirksam sind, keine Therapietreue erfordern und bei Schwangerschaftswunsch beendet, d.h. wieder entfernt werden können.

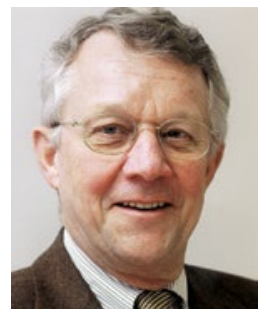

Prof. Dr. med. Heinrich Holzgreve

Internist, Kardiologische Praxis

Burgstr. 7, 80331 München

heinrich.holzgreve@t-online.de 